\title{
Route Optimization with MAP-Based Enhancement in Mobile Networks
}

\author{
Jeonghoon Park, Tae-Jin Lee, and Hyunseung Choo* \\ School of Information and Communication Engineering \\ Sungkyunkwan University \\ 440-746, Suwon, Korea +82-31-290-7145 \\ \{jhpark,tjlee, choo\}@ece.skku.ac.kr
}

\begin{abstract}
The development of wireless network technology and user demands for mobility support have motivated the IETF to introduce mobile IP, mobile IPv6, and its extension, the network mobility (NEMO) basic support protocol. In the NEMO environment, mobile networks form a nested structure. Nested mobile networks based on NEMO basic support (NBS) protocol have the pinball routing problem because packets are routed to all home agents of the mobile routers using nested tunnelling. In this paper, a route optimization scheme is proposed which uses the mobility anchor point employed in hierarchical mobile IPv6, and modifies the binding update messages to minimize overhead in route optimization. We evaluate route optimization cost in terms of delay. The results demonstrate a minimum performance improvement of $30 \%$ and even shorter routing delay than NBS in non-optimized cases.
\end{abstract}

Keywords: Route optimization, routing protocol, mobile networks, pinball routing problem, and mobility anchor point.

\section{Introduction}

As a result of the development of Wireless network technology and demand for mobility support from users, the IETF has introduced mobile IP (MIP) [1, mobile IPv6 (MIPv6) [2], and its extension, the network mobility (NEMO) basic support protocol 3. Route optimization, multi-homing, and security are studied actively in the field of mobile networks that use the NEMO basic support (NBS) protocol. In the NEMO environment, it is assumed that mobile networks can be nested. When the correspondent node $(\mathrm{CN})$ sends a packet to the mobile node (MN), which is located at the nested mobile network, the packet has to visit the home agents (HAs) of all mobile routers (MRs). Further, the packet is tunneled by every HA, because NBS uses a bi-directional tunnel between the MR and HA. This describes the pinball routing problem.

When the packet is routed to all intermediate MR's HA, the routing path becomes too long and the packet size grows due to tunneling, resulting in network inefficiency. And the root-MR or MR's HA link result in a bottleneck for

\footnotetext{
* Corresponding author.
} 
the aggregated traffic from/to all mobile network nodes. If the home network has failures, it cannot ensure the connectivity of mobile networks [4. There have been many research proposals to solve this problem. The HMIP based route optimization method (HMIP-RO) [5] and reverse routing header (RRH) [6] are well-known schemes. HMIP-RO simply extends hierarchical mobile IPv6 (HMIPv6) to the NEMO environment to optimize routing. The RRH proposes an extension of the IPv6 routing header 7. This scheme uses only one tunnel between the MR, which is the nesting MN and MR's HA.

In this paper, we propose a route optimization with MAP-based enhancement (ROME), to solve aforementioned problems in nested mobile networks. ROME uses a mobility anchor point (MAP), which was introduced by HMIPv6 [8, to optimize routing path. This scheme uses a MAP similar to HMIP-RO, but it reduces additional overhead when the MAP is applied to the NEMO environment, by modifying binding update (BU) messages. Hence we evaluate ROME with NBS, when mobile networks use a non-optimized route. When mobile networks optimize its route, the route optimization cost is compared with ROME, HMIP-RO, and RRH, in terms of delay. According to the results, ROME shows a minimum $30 \%$ performance improvement and shorter routing delay than NBS in non-optimized cases.

The subsequent sections of this paper are organized as follows. Section 2 describes a new mechanism for route optimization, named ROME. In Section 3, we evaluate the performance of the ROME scheme, through analytical modelling. This paper is concluded in Section 4.

\section{The Proposed Scheme}

In this section, we propose a new route optimization scheme named ROME. The $\mathrm{CN}$ and MN can find the optimal route using the MAP similar to HMIP-RO.

\subsection{Motivation}

HMIP-RO simply applies the HMIPv6 to the NEMO environment and it has an advantage when mobile networks move within the subnet of the MAP. However, when mobile networks move out of the MAP, a BU storm occurs because all the MRs and MNs in moving mobile networks change their regional care-of address $(\mathrm{RCoA})$ and send BU messages to their HAs. Further, when each MN transmit a packet to the $\mathrm{CN}$, the packet is tunneled as in HMIPv6 whenever it passes through the intermediate MR. This actually induces an additional processing cost in the MRs and accordingly in the MAP.

ROME maintains the advantage of using MAP and resolves the problem of HMIP-RO. It modifies the BU message to avoid the BU storm. The MRs registers the RCoA, home address (HoA), and mobile network prefix (MNP) to their HAs, that is similar to HMIP-RO. Meanwhile, the MNs register the on-link care-of address (LCoA) and HoA to their HAs. When mobile networks move out of the subnet of MAP, only MRs register the RCoA to the HA. Hence, ROME reduces the number of BU messages as many as the number of MNs when mobile 
networks move out of the subnet of MAP. To solve the nested tunnel problem, the MR that receives the router advertisement (RA) message with the MAP option adds the prefix of MAP to the permitted prefix filter range of the ingress filtering option. Accordingly, packets of RCoA source address can pass through the intermediate MRs and the MAP between the MN and the CN.

\subsection{Rome}

In ROME, we assume that the MAP employed in HMIPv6 is a router located in the route aggregation point and requires a certain level of processing ability. The MAP includes its address in the MAP option field of the RA message and propagates it. Similar to the HAs, the MAP manages the network mobility by registering the local $\mathrm{BU}$ messages that is received from the MRs and MNs to its binding cache entry.

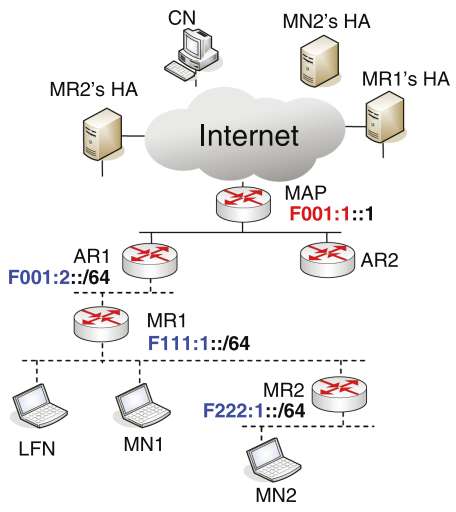

(a) A simple mobile network.

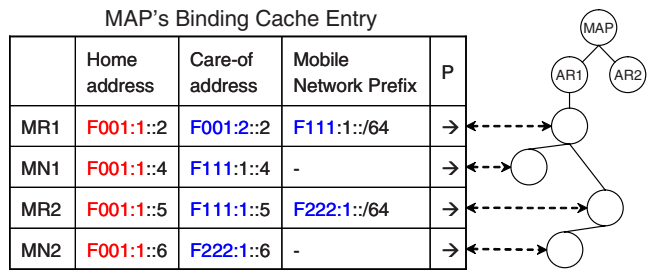

(b) MAP's binding cache entry.

Fig. 1. Basic networks configuration

Network Structure in MAP. The MAP recognizes the mobile network topology by combining BU messages. It manages this topology information using a tree structure, in order to search efficiently, as shown in figures 1 and 2 . The BU messages from the MRs include the MNP of MR as represented in Fig. 2(a). So, The MAP defines these MRs as roots of subtrees as depicted in Fig. 1(b) The MRs and MNs based on this MNP are its children nodes. Based on the subtree, the entire network can be managed by the tree structure as shown in Fig. 1(b) The number of mobile networks using the RCoA with the prefix of the MAP is more than one and even they are overlapped. So, they cannot use duplicate address detection (DAD) [7] for unique address checking. In ROME, the MAP checks the address uniqueness when the MRs or MNs register the RCoA by local $\mathrm{BU}$, and sends the result by binding acknowledgment (BA).

MR Consideration. The MR makes RCoA based on the prefix of the MAP and LCoA based on the prefix of the AR or the MNP of the parent MR. And, it 


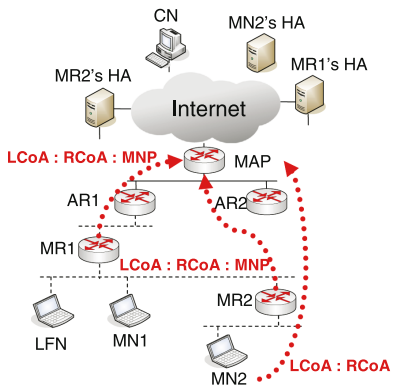

(a) BU for the MAP.

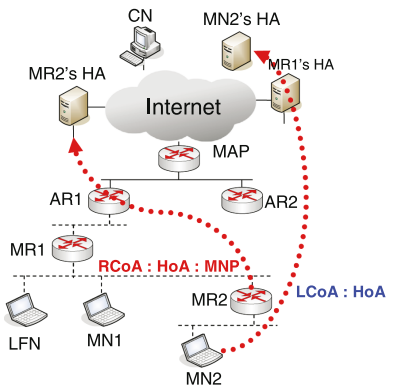

(b) BU for the HA.

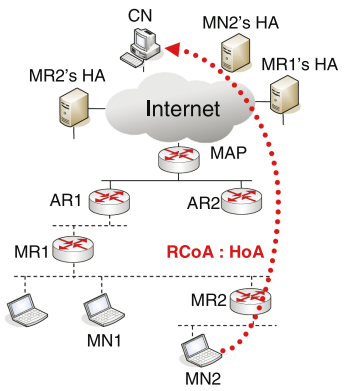

(c) BU for the $\mathrm{CN}$.

Fig. 2. BU messages

appends the prefix of the MAP to the permitted prefix filter range of the ingress filtering option in order for the packets with the RCoA as a source address pass the intermediate MRs and the MAP without tunneling between the MN and CN. After that, the MR registers the RCoA as HoA, and the LCoA as CoA, with the MNP to the MAP, using a local BU, as shown in Fig. 2(a) The MAP can recognize MNs inside the MR by MNP and parent MR by LCoA. Then, the $\mathrm{MR}$ registers the $\mathrm{HoA}$ and $\mathrm{RCoA}$ as $\mathrm{CoA}$ with the MNP to the HA using the $\mathrm{BU}$, as shown in Fig. 2(b). The MR should not register again with the HA when it moves in the subnet of the MAP, because it registers the $\mathrm{RCoA}$, which is the unaffected address in the subnet of the MAP. This reduces BU message cost.

MN Consideration. The MN makes the LCoA using the MNP contained in the RA of MR and the RCoA using the prefix of the MAP contained in the MAP option field. Then, the MN registers the $\mathrm{RCoA}$ as HoA and the LCoA as CoA using the local BU, similar to HMIPv6, as shown in Fig. 2(a). However, the MN registers, unlike HMIP-RO and HMIPv6, LCoA and HoA using BU similar to Mobile IPv6 as depicted in Fig. 2(b). Therefore, when mobile networks move out of the subnet of the MAP, the MN should not perform a BU to the HA. Therefore, a BU storm does not occur. The MN registers the RCoA and HoA to the $\mathrm{CN}$ using a BU as presented in Fig. 2(c). So, the $\mathrm{MN}$ and $\mathrm{CN}$ use the optimal route. If the MN moves in the subnet of the MAP, it should not perform a BU to the $\mathrm{CN}$. This reduces movement registration cost. In addition, ROME guarantees the location privacy of the MNs, since the CNs do not know the LCoA of the MN.

\section{Performance Evaluation}

In this section, we evaluate ROME with existing schemes using two different mechanisms. In the first, we evaluate the non-optimized routing delay in ROME with NBS, because NBS doesn't use an optimal route. In the second, we evaluate route optimization cost in terms of delay between the MN and $\mathrm{CN}$ in ROME, with HMIP-RO and RRH. Table 1 shows the parameters and values used in performance evaluation. 
Table 1. Parameters and Values

\begin{tabular}{l|l|l|l}
\hline Parameter & \multicolumn{1}{|c|}{ Meaning } & \multicolumn{1}{c}{ Value } & Unit \\
\hline \hline$i$ & Nesting level & & \\
\hline$P$ & Propagation speed & $2 \times 10^{8}$ & $\mathrm{~m} / \mathrm{sec}$ \\
\hline$H_{A V G}$ & Average hop between nodes in the wired network & 5 & \\
\hline$D_{A V G}$ & Average distance of a hop in wired networks & $10^{4}$ & $\mathrm{~m}$ \\
\hline$D$ & Average distance of a hop in wireless networks & 10 & $\mathrm{~m}$ \\
\hline$B_{W D}$ & Transmission speed in wired networks & $10^{8}$ & $\mathrm{bit} / \mathrm{sec}$ \\
\hline$B_{W L}$ & Transmission speed in wireless networks & $5.4 \times 10^{7}$ & $\mathrm{bit} / \mathrm{sec}$ \\
\hline$S$ & Normal packet size & $1500 \times 8$ & $\mathrm{bit}$ \\
\hline$S_{T U}$ & Tunnel header size & 320 & $\mathrm{bit}$ \\
\hline$S_{R H}[n]$ & Routing header size with n addresses & $64+128 n$ & $\mathrm{bit}$ \\
\hline$T_{S}$ & Time to search the binding cache & $10^{-4}$ & $\mathrm{sec}$ \\
\hline$T_{C H}$ & Time to change source or destination address & $10^{-4}$ & $\mathrm{sec}$ \\
\hline$T_{R H}$ & Time to process routing header & $5 \times 10^{-4}$ & $\mathrm{sec}$ \\
\hline$T_{T U}$ & Time to process tunnel in entry or exit point & $5 \times 10^{-4}$ & $\mathrm{sec}$ \\
\hline
\end{tabular}

\subsection{Non-optimized Route Delay}

The nodes in mobile networks do not always use the optimal route. For example, the time for the return routability (RR) [2] and BU is considerable overhead when sending small amounts of data. Therefore, mobile networks use the basic protocol, i.e. NBS, and perform route optimization as occasion demands. In this subsection, we compare NBS and ROME when it uses a non-optimized route, in terms of routing delay.

In NBS, the packet from the CN must travel through the MN's HA and all of the MR's HA, and is encapsulated at several levels. However, in ROME, the packet can travel through only the MN's HA and the HA of the MR that belongs to the $\mathrm{MN}$, and it is encapsulated at two levels. In general, the total delay of the packet consists of transmission delay, propagation delay, and processing delay. Represented as follows.

$$
D_{\text {Total }}=D_{\text {Trans }}+D_{\text {Prop }}+D_{\text {Proc }}
$$

Fig. 31 represents the example of route delay in NBS and ROME. Transmission delay, propagation delay, and processing delay in NBS generalized by nesting level are derived as follows.

$$
\begin{aligned}
& D_{N B S-\text { Trans }}=\frac{(i+2) S+\sum_{k=1}^{i+1} k \cdot S_{T U}}{B_{W D}} \cdot H_{A V G}+\frac{(i+1) S+\sum_{k=1}^{i+1} k \cdot S_{T U}}{B_{W L}}, \\
& D_{N B S-\text { Prop }}=\frac{(i+2) D_{A V G}+(i+1) D}{P}, \\
& D_{N B S-\text { Proc }}=(i+1) T_{S}+2(i+1) T_{T U} .
\end{aligned}
$$

Transmission delay, propagation delay, and processing delay in ROME generalized by nesting level are derived as follows. 


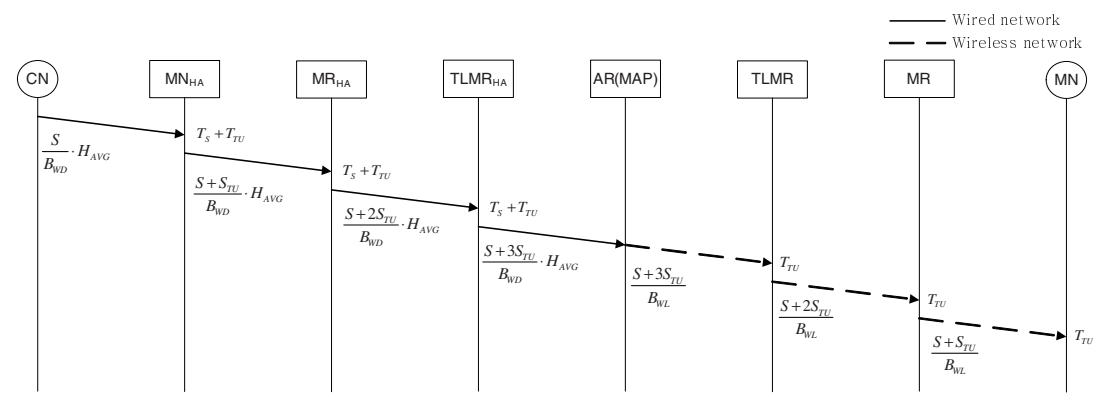

(a) Packet delay for the NBS.

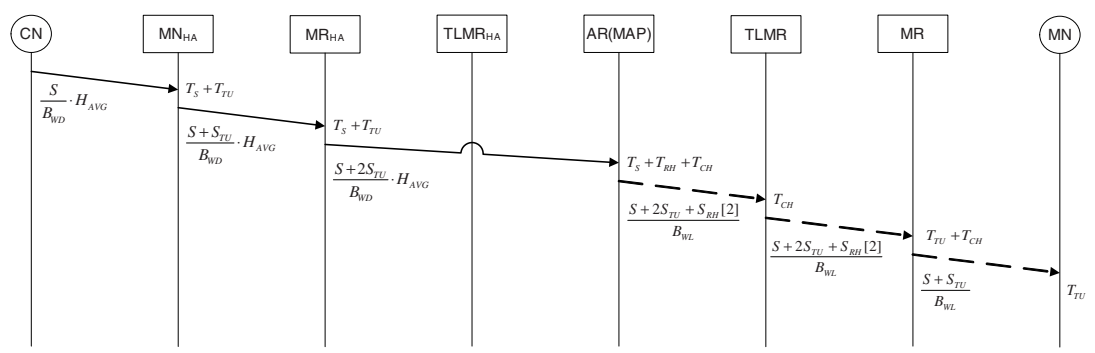

(b) Packet delay for ROME.

Fig. 3. Packet delay for the NBS and ROME

$$
\begin{aligned}
& D_{R O M E-T r a n s}=\frac{3 S+3 S_{T U}}{B_{W D}} \cdot H_{A V G}+\frac{(i+1) S+(2 i+1) S_{T U}+i \cdot S_{R H}[i]}{B_{W L}}, \\
& D_{R O M E-\text { Prop }}=\frac{3 D_{A V G}+(i+1) D}{P} \\
& D_{R O M E-\text { Proc }}=3 T_{S}+4 T_{T U}+T_{R H}+(i+1) T_{C H} .
\end{aligned}
$$

Fig. 4 represents the performance result of comparison between NBS and ROME. ROME shows lower performance than NBS, when the nesting level is 1 , because the packet routing path is the same and the packet is encapsulated the same number of times, but ROME attaches the routing header for source routing in the MAP. ROME shows higher performance than NBS when the nesting level is 2 or greater. In particular, ROME shows greater than $60 \%$ performance improvement when the nesting level is 10 .

\subsection{Delay for Route Optimization}

The route optimization schemes in the NEMO environment perform route optimization when the MN receives the encapsulated packet from its HA. In this subsection, we compare the route optimization cost of the HMIP-RO, RRH, and ROME in terms of delay.

The delay for route optimization in HMIP-RO is generalized by the nesting level derived as follows. 


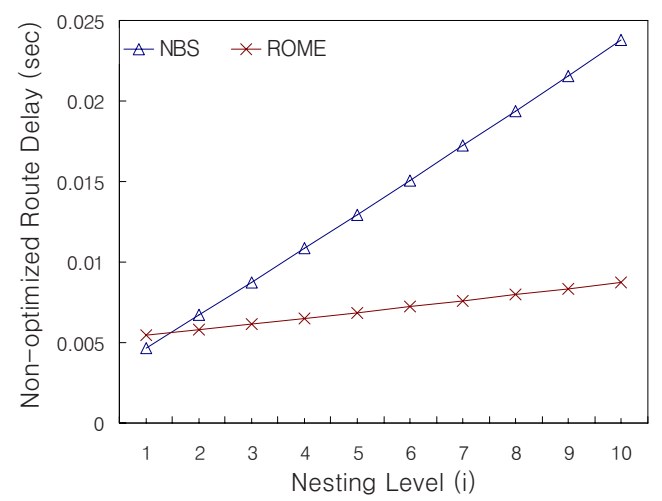

Fig. 4. Comparison of non-optimized route delay

$$
\begin{aligned}
R O D_{H M I P-T r a n s}[i] & =\frac{4 S+3 S_{T U}}{B_{W D}} \cdot H_{A V G} \\
& +\frac{3(i+1) S+\left\{\sum_{k=1}^{i+1} k+(i+1)\right\} \cdot S_{T U}+2 S_{R H}[i+1]}{B_{W L}}, \\
\operatorname{ROD}_{H M I P-P r o p}[i]= & \frac{4 D_{A V G}+3(i+1) D}{P}, \\
\operatorname{ROD}_{H M I P-P r o c}[i]= & 3 T_{S}+2(i+2) T_{T U}+2 T_{R H}+2(i+1) T_{C H} .
\end{aligned}
$$

Transmission delay, propagation delay, and processing delay for route optimization in RRH are generalized by nesting level, as follows.

$$
\begin{aligned}
& R O D_{R R H-T r a n s}[i]=\frac{(i+6) S+2 S_{R H}[i+1]+\left(\sum_{k=1}^{i+1} k+2\right) S_{T U}}{B_{W D}} \cdot H_{A V G} \\
&+\frac{3(i+1) S+\left(\sum_{k=1}^{i+1} k+2\right) S_{T U}+2 i \cdot S_{R H}[i+1]}{B_{W L}}, \\
& R O D_{R R H-P r o p}[i]=\frac{(i+6) D_{A V G}+3(i+1) D}{P}, \\
& R O D_{R R H-\text { Proc }}[i]=(i+2) T_{S}+2(i+3) T_{T U}+2 T_{R H}+2(i-1) T_{C H} .
\end{aligned}
$$

The transmission delay, propagation delay, and processing delay for route optimization in ROME generalized by nesting level are derived as follows.

$$
\begin{aligned}
& R O D_{R O M E-T r a n s}[i]=\frac{5 S+3 S_{T U}}{B_{W D}} \cdot H_{A V G} \\
&+\frac{3(i+1) S+(2 i+1) S_{T U}+2(i+1) S_{R H}[i+1]}{B_{W L}}, \\
& R O D_{R O M E-P r o p}[i]=\frac{5 D_{A V G}+3(i+1) D}{P} \\
& R O D_{R O M E-P r o c}[i]=4 T_{S}+5 T_{T U}+2 T_{R H}+2(i+1) T_{C H} .
\end{aligned}
$$

Fig. 5 shows the total route optimization delay for each scheme using Table 1 . ROME shows at least $30 \%$ improvement over the other schemes. 


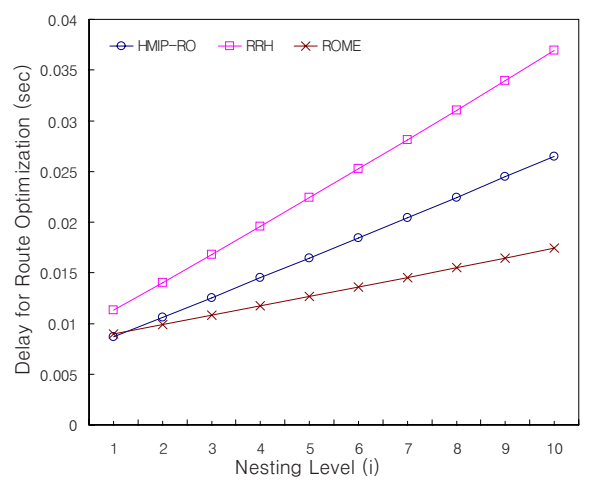

Fig. 5. Route optimization delay in various nesting level

\section{Conclusion}

In this paper, we propose a route optimization scheme based on the MAP, similar to HMIP-RO. ROME exhibits a shorter route length than the NBS, when mobile networks do not perform the route optimize procedure. The proposed scheme does not use tunneling between the MN and MAP, unlike HMIP-RO. Therefore, it reduces the overhead caused by tunneling. The BU storm is reduced by modifying the BU messages for the HA. The performance of the proposed scheme is better than existing schemes, HMIP-RO and RRH. In the future, we will study not only inter-NEMO route optimization, but also intra-NEMO route optimization, in mobile networks.

Acknowledgment. This research was supported by MIC, Korea under ITRC IITA-2006-(C1090-0603-0046)

\section{References}

1. Perkins, C.: IP Mobility Support for IPv4. RFC 3344, IETF (2002)

2. Johnson, D., Perkins, C., Arkko, J.: Mobility Support in IPv6. RFC 3775, IETF (2004)

3. Devarapalli, V., Wakikawa, R., Petrescu, A., Thubert, P.: Network Mobility (NEMO) Basic Support Protocol. RFC 3963, IETF (2005)

4. Ng, C., Thubert, P., Watari, M., Zhao, F.: Network Mobility Route Optimization Problem Statement. Internet draft, IETF (2006)

5. Ohnishi, H., Sakitani, K., Takagi, Y.: HMIP based Route Optimization Method in A Mobile Network. Internet draft, IETF (2003)

6. Thubert, P., Molteni, M.: IPv6 Reverse Routing Header and Its Application to Mobile Networks. Internet draft, IETF (2004)

7. Deering, S., Hinden, R.: Internet Protocol, Version 6 (IPv6). RFC 2460, IETF (1998)

8. Soliman, H., Castelluccia, C., El-Malki, K., Bellier, L.: Hierarchical Mobile IPv6 Mobility Management (HMIPv6). RFC 4140, IETF (2005)

9. IETF Network Mobility (NEMO) Working Group, IETF. 